\title{
Prospects for SME Tests with Experiments at SYRTE and LKB
}

\author{
C. Guerlin, ${ }^{1,2}$ H. Pihan-Le Bars, ${ }^{2}$ Q.G. Bailey, ${ }^{3}$ and P. Wolf ${ }^{2}$ \\ ${ }^{1}$ Laboratoire Kastler Brossel, ENS-PSL Research University, CNRS, \\ UPMC-Sorbonne Universités, Collège de France, 75005 Paris, France \\ ${ }^{2}$ SYRTE, Observatoire de Paris, PSL Research University, CNRS, \\ Sorbonne Universités, UPMC, 75014 Paris, France \\ ${ }^{3}$ Embry-Riddle Aeronautical University, Prescott, Arizona 86301, USA
}

\begin{abstract}
Preliminary work has been done in order to assess the perspectives of metrology and fundamental physics atomic experiments at SYRTE and LKB in the search for physics beyond the Standard Model and General Relativity. The first studies we identified are currently ongoing with the Microscope mission and with a Cs fountain clock. The latter brings significant improvement on the proton-sector coefficient $\bar{c}_{T T}$ down to the $10^{-17} \mathrm{GeV}$ level.
\end{abstract}

\section{Experiments and SME sectors of interest}

We investigate the use of atomic experiments and Earth orbit space missions at SYRTE and LKB in the search for Lorentz-invariance violations (LIV) in the framework of the Standard-Model Extension (SME). We worked, mainly in the minimal SME, on gathering from the literature LIV effects, models, and perspectives for the experiments considered (Secs. 2 and 3). We identified the most promising and feasible experiments and data analysis, which is currently work in progress (Sec. 4). The experiments considered include: the double-species Cs fountain clock (FO2), the cold atom gravimeter (CAG), the superconducting gravimeter, the GBAR experiment, ${ }^{1}$ the ACES mission, ${ }^{2}$ and the Microscope Weak Equivalence Principle (WEP) test mission. ${ }^{3}$ The SME models for these experiments involve the fermion, gravity, and photon sectors. We focused on the matter CPT-even $\bar{c}_{\mu \nu}^{w}$ tensor and CPT-odd $\bar{a}_{\mu}^{w}$ vector $(w=e, p, n)$, on disentangling them, and on pushing constraints towards or beyond their expected Planck scale suppression, i.e., $10^{-19} \mathrm{GeV}$. 


\section{SME LIV in center of mass motion}

Including gravitational-sector LIV with matter-gravity couplings in a classical treatment modifies the classical dynamics of massive bodies. As shown in Eq. (132)-(134) in Ref. 4, the effective inertial mass of test bodies gets tensor components due to $\bar{c}_{\mu \nu}^{w}$, while $\bar{c}_{00}^{w}$ and $\bar{a}_{0}^{w}$ modify the gravitational force. It results in isotropic and frame-dependent LIV modifications to the effective acceleration. The isotropic part is $g^{T}=g\left(1+\beta^{T}\right)$ with $\beta^{T}=\frac{2 \alpha}{m^{T}}\left(\bar{a}^{T}\right)_{0}-\frac{2}{3}\left(\bar{c}^{T}\right)_{00}$ for a test mass $m^{T}$. Due to a change of the $\bar{a}_{0}^{w}$ sign for antimatter, free-fall LIV could still be large for antimatter while strongly constrained for normal matter, ${ }^{5}$ as can be measured, e.g., in GBAR in the future (free fall of antihydrogen). ${ }^{1}$ Deriving the equations of motion from the lagrangian by the Euler-Lagrange equations and expressing frame dependence with respect to a common inertial frame (the Sun-centered celestial equatorial frame) leads to the full LIV time variation model, which we can use to model the observables of gravimeters and WEP tests. ${ }^{4}$ Note that for any neutral test body, the proton to electron SME coefficient ratio is fixed in this type of test.

\section{SME LIV in atomic internal energy}

Atoms are composite systems in quantized bound states. SME LIV energy shifts can be calculated perturbatively from the nonrelativistic free-fermion hamiltonian. Spin-independent contributions can be classified in LIV and metric fluctuation order as ${ }^{4} H=H^{(0,1)}+H_{c}^{(1,1)}$.

The first term describes LIV shifts for clocks in flat spacetime, ${ }^{6}$ arising as an anisotropy of the fermionic dispersion relation. Indeed nonzero LIV expectation values arise for each particle from the operator $\delta H=$ $-\mathcal{C}_{0}^{(2)} \mathcal{P}_{0}^{(2)} / 6 m$ (in spherical tensor notation) which involves the quadrupole moment of $\bar{c}_{\mu \nu}^{w}$ and $p_{i} p_{j}$ tensors: $\mathcal{C}_{0}^{(2)}=\bar{c}_{j j}-3 \bar{c}_{33}=\bar{c}_{Q} / m$ and $\mathcal{P}_{0}^{(2)}=$ $\boldsymbol{p}^{2}-3 p_{3}^{2}$ in cartesian coordinates with the laboratory frame axis 3 along the quantization axis. Analysis of sidereal variations in magnetized Zeeman substates, either by measuring hyperfine frequency in a Cs fountain clock $^{7}$ or Larmor precession in co-magnetometers, ${ }^{8}$ has allowed constraining proton and neutron anisotropic $\bar{c}_{\mu \nu}^{w}$ coefficients beyond the Planck scale suppression. The analysis is in fact nucleus model dependent, which is currently being investigated. ${ }^{9}$ For electrons, the best sensitivity has been reached with dysprosium ${ }^{10}$ and $\mathrm{Ca}^{+}$ions ${ }^{11}$ spectroscopy.

The second term describes LIV modification in curved spacetime. It is smaller since of overall second order but relevant for constraining the 
isotropic $\bar{c}_{T T}^{w}$ coefficients, otherwise suppressed by boosts and often dismissed in analyses. This term is $\delta H=\left(2 U / 3 c^{2}\right) \bar{c}_{00}^{w} \boldsymbol{p}^{2} / 2 m$ with $U$ the newtonian gravitational potential. It can be seen as a potential-dependent rescaling of electron and proton inertial masses, leading to modified binding energies and thus to a modified clock gravitational redshift. Redshift measurement on clocks as well as null redshift tests, in the gravitational field of Earth and Sun, thus constrain proton and electron $\bar{c}_{T T}^{w}$ coefficients. ${ }^{4,12}$ This brings a competitive limit for the latter one at $10^{-8} \mathrm{GeV} .{ }^{10}$

Note that within the SME, WEP violations and modified reshift come together, both due to species dependent LIV. In addition, WEP violation is also slightly modified by internal energy LIV. Indeed nuclear binding energy contributes to up to $1 \%$ of the atom's rest mass, so LIV modification to nuclear binding energy also modifies the free fall of the atom. ${ }^{12,13}$ This helps disentangling electron from proton contributions.

\section{Progress and perspectives}

For gravimeter experiments, our progress so far concerns the model and systematics evaluation. Combining Refs. 4 and 14, we derived the gravimeter model including the Earth's spherical inertia $i_{E}, \bar{c}_{\mu \nu}^{w}, \bar{a}_{\mu}^{w}$ and $\bar{s}_{\mu \nu}$, to order $V_{L}, V_{E}$ (laboratory and Earth boost). This improved form has been used in Ref. 15 in Eq. (21). We then questioned whether tidal models used in gravimeters subtract SME signatures. Indeed tidal models are usually fitted to data related to free fall, while all SME frequencies overlap with tidal frequencies. ${ }^{16}$ To our knowledge this issue has not been addressed yet in detail in the literature for gravimeter tests. We are currently working along this direction. Progress of the performances of atom gravimeters since the analysis in Ref. 17 could give interesting improved constraints, as well as analyzing the longer time series that are available from geophysics observations (this approach was independently mentioned and a preliminary discussion given by J.D. Tasson. ${ }^{18}$ )

For the spin-polarized Cs fountain clock, keeping the second order boost suppressed terms for all $\bar{c}_{\mu \nu}$ coefficients, we reanalyzed the data presented in Ref. 7. We made only few assumptions in our model and treatment, keeping track of all correlations present in our data, and calculated confidence intervals. This allowed us to constrain the proton $\bar{c}_{T T}$ coefficient, in the Schmidt model, down to the $10^{-17} \mathrm{GeV}$ scale, improving present limits by six orders of magnitude compared to WEP tests, ${ }^{4,19}$ bringing it close to the Planck scale suppression. More details will be given in a dedicated 
article. $^{20}$

The Microscope mission was launched on April 25, 2016, aiming for improving the WEP test ${ }^{3}$ down to $10^{-15}$. Based on Ref. 4, a proposal for SME analysis has been accepted by CNES and the Science Working Group. We are currently investigating the calibration procedure of systematic effects in order to identify at which data treatment level we should proceed with an SME analysis without losing possible LIV signals. We expect this mission to set the best limits on all $\bar{a}_{\mu}^{w}$ coefficients down to $10^{-13} \mathrm{GeV}$ (isotropic coefficient), improving present constraints by three to six orders of magnitude. ${ }^{15,19}$

Beyond these tests, we are performing simulations of spin polarized states LIV on ACES. ${ }^{2}$ Work on nuclear models is also being pursued.

\section{Acknowledgments}

C.G., Q.G.B., and P.W. acknowledge support from the Sorbonne Universités grant Emergence for the CABESTAN collaboration. Q.G.B. acknowledges the National Science Foundation under grant number PHY-1402890.

\section{References}

1. P. Perez and Y. Sacquin, Class. Quantum Grav. 29, 184008 (2012).

2. P. Laurent et al., C.R. Physique 16, 540 (2015).

3. P. Touboul et al., Acta Astronautica 50, 433 (2002).

4. V.A. Kostelecký and J.D. Tasson, Phys. Rev. D 83, 016013 (2011).

5. J.D. Tasson, Hyperfine Int. 228, 111 (2014).

6. V.A. Kostelecký and C.D. Lane, Phys. Rev. D 60, 116010 (1999); R. Bluhm et al., Phys. Rev. D 68, 125008 (2003).

7. P. Wolf et al., Phys. Rev. Lett. 96, 060801 (2006).

8. M. Smiciklas et al., Phys. Rev. Lett. 107, 171604 (2011).

9. B.A. Brown et al., arXiv:1604.08187; V.V. Flambaum, arXiv:1603.05753.

10. M.A. Hohensee et al., Phys. Rev. Lett. 111, 050401 (2013).

11. T. Pruttivarasin et al., Nature 517, 592 (2015).

12. M.A. Hohensee et al., Phys. Rev. Lett. 106, 151102 (2011).

13. M.A. Hohensee et al., Phys. Rev. Lett. 111, 151102 (2013).

14. Q.G. Bailey and V.A. Kostelecký, Phys. Rev. D 74, 045001 (2006).

15. A. Hees et al., Phys. Rev. D 92, 064049 (2015).

16. Y. Tamura, Bull. Inf. Marées Terrestres 99, 6813 (1987).

17. K.Y. Chung et al., Phys. Rev. D 80, 016002 (2009).

18. J.D. Tasson, these proceedings.

19. Data Tables for Lorentz and CPT Violation, V.A. Kostelecký and N. Russell, 2016 edition, arXiv:0801.0287v9.

20. H. Pihan-Le Bars et al., in preparation. 